\title{
Applying QMSIW Technique in Textile for Compact Wearable Design and high Body-Antenna Isolation
}

\author{
Sam Agneessens, Sam Lemey, Hendrik Rogier \\ Department of Information Technology (INTEC) \\ Ghent University \\ Ghent, Belgium \\ email: sam.agneessens@intec.ugent.be
}

\author{
Thomas Vervust, Jan Vanfleteren \\ Department of Electronics and Information Systems (ELIS) \\ Ghent University \\ Ghent, Belgium
}

\begin{abstract}
The Quarter-Mode Substrate Integrated Waveguide technology is applied in textile materials to obtain an antenna small in dimensions, simple in design, and with excellent bodyantenna isolation. The design evolution is presented, and a prototype is fabricated and validated. Measured on-body bandwidth of $5.1 \%$ and gain of $4.2 \mathrm{dBi}$ are achieved. Due to the good shielding a very low Specific Absorption Rate of $0.45 \mathrm{~mW} / \mathrm{g}$, averaged over $1 \mathrm{~g}$ of tissue, for $500 \mathrm{~mW}$ input power is obtained.
\end{abstract}

\section{INTRODUCTION}

Wearable systems have an enormous potential, including remote telemetry, augmented reality, vital sign monitoring of rescue workers. These wireless systems rely on batteries or energy harvesting for power and should be designed as efficiently as possible. As such, a good dedicated antenna design is indispensible.

Textile antennas are very popular in wearable applications as they are fabricated from the same material as the wearer's garments. Moreover, they are easily integrated inconspicuously into the clothing. Most of these designs are implemented in microstrip technology and offer excellent performance in free space and on-body. They achieve these good on-body results by relying on a ground plane to effectively shield the wearer from the antenna and vice versa, resulting in stable -and most importantly - efficient on-body performance. For the ground plane to effectively shield the wearer from radiation, its size should extend sufficiently beyond the patch's size, which results in larger overall antenna dimensions.

To overcome this issue other implementation techniques can be explored, such as Substrate Integrated Waveguide (SIW) techniques, which use rows of conductive posts to implement rectangular waveguides in planar from. Owing to the effective confinement of EM-fields in the structure by the vias there is no longer need for a large ground plane. The technique also allows miniaturization of the design by taking advantage of the symmetry in the EM-field distribution of the antenna's resonant modes [1]. Recently the SIW implementations have been realized on textile materials, using eyelets [2] or conductive thread to implement the vias [3].

The design presented here is a circular quarter-mode SIW antenna which relies on two symmetry planes in the mode distribution of a circular resonant cavity and additional miniaturization slots to achieve a compact antenna with very robust on-body performance.

\section{QMSIW WEARABLE ANTENNA DESIGN \& FABRICATION}

The design evolution is visualized in Fig. 1. The starting point is a circular resonant cavity fed by a coaxial probe. This structure's dimensions are reduced by cutting away the top half along the symmetry planes of its resonant mode, which act as a virtual magnetic wall. This is done twice (along the XZ-plane and the YZ-plane), resulting in a cavity operating at $\sim \lambda / 4$. Consequently, radiation leakage occurs through the open side walls and the structure starts to operate as an effective antenna with boresight along the z-axis, directed away from the wearer. Additional slots are added to reduce the resonance frequency, leading to in a further size reduction. This straightforward design evolution results in a highly wearable, small, and simple textile antenna topology.

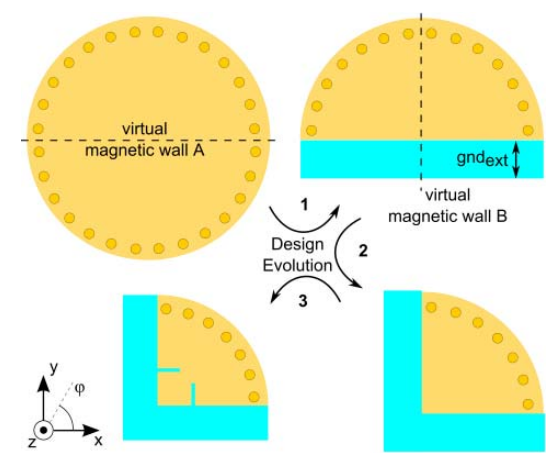

Fig. 1. Design evolution: the symmetry of the first resonant mode is exploited in the first two steps, in addition to current displacing slots added in the final step, to significantly reduce the dimensions while maintaining high body-antenna isolation.

The proposed final geometry, shown in Fig. 2, is developed for communication between a wearable system and an off-body terminal in the $2.4 \mathrm{GHz}$ Industrial Scientific Medical (ISM) band. The design is implemented in SIW technology on textile: brass eyelets (diameter, $\mathrm{d}=4 \mathrm{~mm}$, and spacing, $\mathrm{s}=8 \mathrm{~mm}$ ) are fixed with a hand-press on a wearable rubber substrate commonly found in firefighter gear $(\mathrm{h}=3.7 \mathrm{~mm}$, $\mathrm{\varepsilon r}=1.495$, 
$\tan \delta=0.016$ ), and conductive e-textile copper-plated polyester taffeta fabric (thickness $80 \mu \mathrm{m}$ and surface resistivity $0.18 \Omega / \mathrm{sq}$ at $2.45 \mathrm{GHz}$ ) is used to realize the conductive layers. The conductive sheets are patterned by laser cutting with a picosecond pulsed laser with a spotsize of $20 \mu \mathrm{m}$, resulting in very accurate fabrication dimensions.

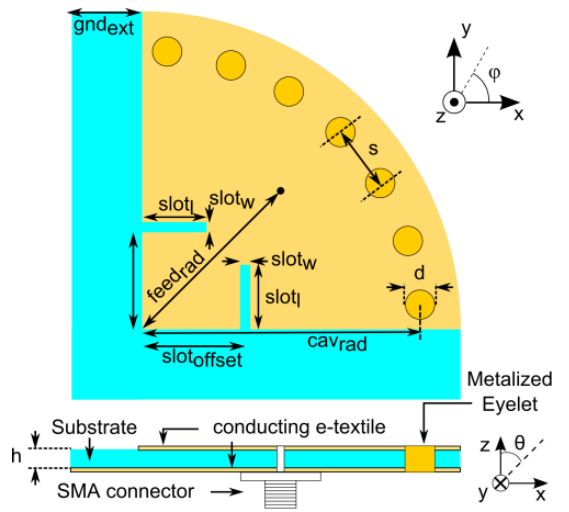

Fig. 2. Wearable Quarter-Mode SIW textile antenna topology. $\mathrm{cav}_{\mathrm{rad}}=34.9 \mathrm{~mm}$, slot $_{1}=8 \mathrm{~mm}$, slot $_{\mathrm{w}}=1 \mathrm{~mm}$, slot $_{\text {offset }}=12.9$ $\mathrm{mm}$, feed $_{\mathrm{rad}}=17.4 \mathrm{~mm}$, gnd $_{\mathrm{ext}}=25 \mathrm{~mm}, \mathrm{~d}=4 \mathrm{~mm}, \mathrm{~s}=8 \mathrm{~mm}$.

\section{DESIGN VALIDATION}

Optimization and fine tuning of the design are performed with CST microwave studio. Validation of the design is done by comparing the simulated and measured impedance matching and radiation characteristics for different scenarios, being free space and an on-body configuration.

Fig. 3 shows the simulated and measured reflection coefficient, w.r.t. $50 \Omega$, in a free space and on-body scenario. Good agreement can be observed for all cases, indicating robust on-body performance. The measured fractional bandwidth in free space is $4.8 \%$ and $5.1 \%$ when measured onbody, which is a slight increase, owing to additional losses caused by the human body. The center resonant frequency, however, stays stable despite the proximity of the human body.

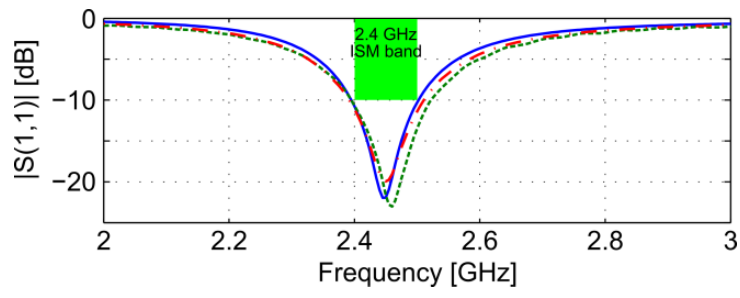

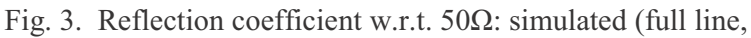
blue), measured (dot-dashed line, red), and on-body measured (dotted line, green).

Cross-sections of the gain patterns in the E-plane $\left(\varphi=45^{\circ}\right)$ and H-plane $\left(\varphi=135^{\circ}\right)$, for the different scenarios (free space: simulation \& measurement, on-body: measurement) are shown in Fig. 4. The measured and simulated free-space results agree very well, both in terms of realized gain value and and in terms of pattern shape. A maximum simulated and measured gain of $4.4 \mathrm{dBi}$ and $4.2 \mathrm{dBi}$, respectively, are obtained, in addition to a simulated radiation efficiency of $76 \%$ versus a measured value of $81 \%$.
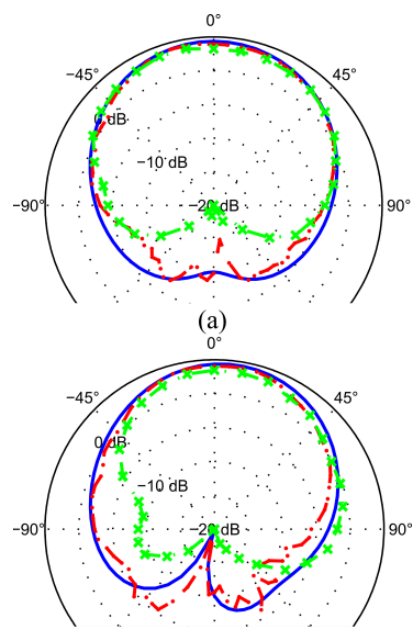

(b)

Fig. 4. Antenna radiation pattern in the (a) E-plane $\left(\varphi=45^{\circ}\right)$ and (b) H plane $\left(\varphi=135^{\circ}\right)$ : free space, simulated (full line,

blue) and measured (dot dashed red line); on-body measurement (dotted green line, $\mathrm{x}$-marker)

An estimated on-body radiation efficiency of $60 \%$ was achieved in simulations when the antenna was placed at $2 \mathrm{~mm}$ distance from the human body. This corresponds to a $1 \mathrm{~dB}$ loss in radiation efficiency due to body absorption. The Specific Absorption Rate of $0.45 \mathrm{~mW} / \mathrm{g}$ was calculated for $500 \mathrm{~mW}$ input power averaged over $1 \mathrm{~g}$ of human tissue.

These good on-body results both in terms of impedance matching and radiation performance (efficiency \& SAR) can be attributed to the dedicated design, which aims at high-body antenna isolation by relying on a ground extension and a directive radiation pattern.

\section{REFERENCES}

[1] C. Jin, R. Li, A. Alphones and X. Bao, "Quarter-Mode Substrate Integrated Waveguide and Its Application to Antennas Design," Antennas and Propagation, IEEE Transactions on, vol. 61, no. 6, pp. 2921-2928, June 2013.

[2] S. Agneessens and H. Rogier, "Compact Half Diamond Dual-Band Textile HMSIW On-Body Antenna," Antennas and Propagation, IEEE Transactions on, vol. 62, no. 5, pp. 2374-2381, May 2014.

[3] T. Kaufmann and C. Fumeaux, "Wearable Textile Half-Mode SubstrateIntegrated Cavity Antenna Using Embroidered Vias," Antennas and Wireless Propagation Letters, IEEE, vol. 12, pp. 805-808, 2013. 